\title{
Anomalous diffusion in a variable area whose boundary moves with a constant speed
}

\author{
Xicheng Li \\ School of Mathematical Science, University of Jinan, Jinan, P.R. China \\ xichengli@yahoo.com.cn
}

\begin{abstract}
In this paper, we study a space-fractional anomalous diffusion in a variable area. The moving boundary is assumed moving with constant speed. The numerical scheme was present by changing the moving boundary to a fixed one. The steady-state approximation was also given to show the properties of the diffusion process.
\end{abstract}

Keywords-fractional calculus; moving boundary; steady state approximation; anomalous diffusion

\section{Introduction}

Recently, the fractional differential equations [1,2] has been used to model physical and engineering processes. The fractional anomalous diffusion equation is perhaps the most frequently studied complex problem. Classical partial differential equation of diffusion and wave equation has been extended to the equation with fractional time or space by means of fractional operators. In a normal diffusion process, the flux can be written as

$$
q=K \frac{\partial c(x, t)}{\partial x}
$$

where $c(x, t)$ is the concentration of the solute and $K$ is the diffusion coefficient. A generalization of the flux is changing it to a fractional one as $[3,4]$

$$
q=K \frac{\partial^{\alpha-1} c(x, t)}{\partial x^{\alpha-1}}, \quad 1<\alpha<2,
$$

where $\frac{\partial^{\beta}}{\partial x^{\beta}}$ is a space fractional derivative in the Riemman -Liouville sense, Caputo sense, Riesz-Feller sense or others.

In this study, we will use the Caputo type fractional derivative as the space derivative operator. The $\beta$-th order fractional derivative of $f(x)$ is defined as

${ }_{0}^{c} D_{x}^{\beta} f(x)=\frac{1}{\Gamma(n-\beta)} \int_{0}^{x}(x-\xi)^{n-\beta-1} \frac{\mathrm{d}^{n} f(\xi)}{\mathrm{d} \xi^{n}} \mathrm{~d} \xi$,

where $n$ is an integer such that $n=[\beta]+1$. Using the constitutive equation, the fractional diffusion equation can be written as

$$
\frac{\partial c(x, t)}{\partial t}=K_{0}^{C} D_{x}^{\alpha} c(x, t), \quad 1<\alpha \leq 2 .
$$

Several analytical methods have been used to solve fractional differential equations, such as the integral transform methods, Adomian decomposition method and other methods. To solve many science and engineering problems, the numerical solutions of fractional differential equations also attract many attentions [5]. The research on the numerical methods of fractional equations is by far less developed and understood than its non-fractional counterpart. The first numerical algorithm is the Grünwald-Letnikov one which is often used to numerically approximate the Riemman-Liouville fractional derivative. For the initial value problems, by using the Volterra integral equations equal to the original fractional equations, Diethelm et al. [6,7] presented a numerical approximation using Adams type predictor corrector approach and gave the corresponding detailed error analysis. To reduce the computational cost, Ford and Simpson [8] presented the nested memory concept which can lead to $O\left(h^{-1} \log \left(h^{-1}\right)\right)$ complexity. Deng [9] apprehended the short memory principle and extended the range from $\alpha \in(0,1)$ to $\alpha \in(0,2)$. More recently, $\mathrm{Li}$ [10] generalized the B-spline collection method to fractional differential equations. Most studies on the fractional anomalous diffusion equation are in fixed areas. In 2007, Liu and $\mathrm{Xu}$ [11] firstly presented an analytical solution to the moving boundary problem of anomalous diffusion arisen in controlled drug release system. Li et al. [12] studied a space-time fractional moving boundary problem in which the space fractional derivative was in the Riesz-Feller sense. In another paper [13], they gave the similarity solutions to the time-space fractional moving boundary problem when the space fractional derivatives are in the Caputo sense or the RiemmanLiouville sense.

The author is supported by the Natural Science Foundation of China (N0. 11002049) and the Shangdong Province Young and MiddleAged Scientists Research Awards Fund (No. BS2012SW002). 
In this paper, we will consider a moving boundary problem in which one of the boundaries is moving with constant speed. The paper is organized as follows. In section 2 , the mathematic model is presented and some analysis is given. In section 3 and section 4 , the numerical solution of the model and some discussions are given. Finally in section 5, the conclusions are presented.

\section{Mathematical model and analysis}

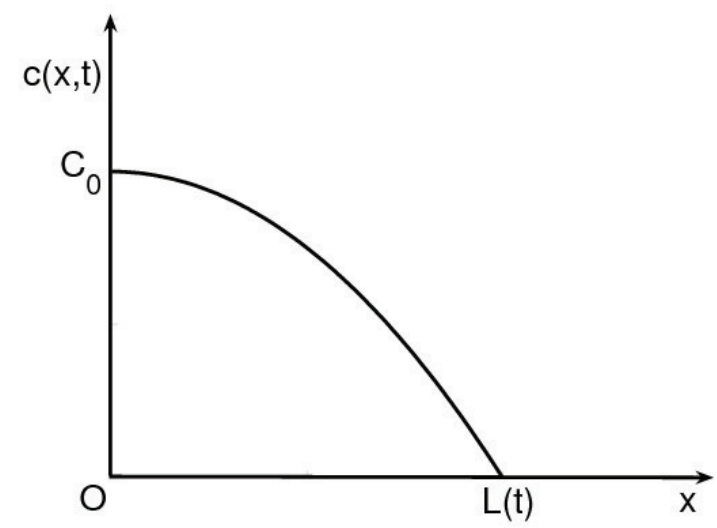

Figure 1. Illustration of the concentration at time $t$.

The concentration at time $t$ is illustrated in Fig. 1 .

Assume that the diffusion area is $0 \leq x \leq L(t)$,

where $L(t)$ is a function of $t$, i.e., one of the boundary is moving as $t$ progressing. Using the fractional diffusion equation (4) as the governing equation, i.e.,

$$
\frac{\partial c(x, t)}{\partial t}=K_{0}^{C} D_{x}^{\alpha} c(x, t), \quad 0 \leq x \leq L(t), t>0 .
$$

A constant source and a perfect sink are placed at $x=0$ and $x=L(t)$. If we use the assumption that the moving boundary moving with a constant speed, the boundary condition can be written as

$$
\begin{array}{cc}
c(0, t)=C_{0}, & t>0, \\
(6) & \\
c(L(t), t)=0, & t>0, \\
(7) & (L(t)=m t) .
\end{array}
$$

If the governing equation is of integer order, this problem can be handled by many methods because there are kinds of variable transforms can be used. However, it is not the case for the fractional diffusion equation for the reason that many useful properties of the ordinary derivative are not known to carry over analogously for the case of fractional derivatives, such as a clear geometric mean, the product rules, chain rules and so on. For example, the Leibniz rule for evaluating the $n$-th derivative of the product $\psi(t) f(t)$ is

$\frac{\mathrm{d}^{n}}{\mathrm{~d} t^{n}}(\psi(t) f(t))=\sum_{k=0}^{n} C_{n}^{k} \psi^{(k)}(t) f^{(n-k)}(t)$,

(9)

but the Leibniz rule for the fractional derivative takes the form

$$
{ }_{0} D_{t}^{p}(\psi(t) f(t))=\sum_{k=0}^{\infty} C_{p}^{k} \psi^{(k)}(t)_{a} D_{t}^{p-k} f(t) .
$$

It is a infinite series and difficult to use.

From the mathematical point of view, the moving boundary problems are difficult to obtain their analytical solutions. We will firstly give a numerical scheme of the calculation of the model.

\section{Numerical solution}

Though the governing equation and the boundary conditions seem to be simple, due to the moving boundary, its numerical scheme is also difficult to given. In order to simplify the problem, we change the variable area to a fixed one using the following transforms

$$
z=\frac{x}{L(t)}, \quad C=\frac{c}{C_{0}} .
$$

The reduced problem is

$$
m^{\alpha} t^{\alpha} \frac{\partial C(z, t)}{\partial t}-m^{\alpha} t^{\alpha-1} z \frac{\partial C}{\partial z}=K_{0}^{C} D_{z}^{\alpha} C(z, t), \quad 0 \leq z \leq
$$

$$
\begin{array}{ll}
C(0, t)=1, & t>0, \\
(13) & \\
C(1, t)=0, & t>0 .
\end{array}
$$

If we use $C_{j}^{k}$ denote the concentration at the position $z=k h$ and the time $t=k \Delta t$, the forward difference scheme of the first order derivatives are

$$
\frac{\partial C}{\partial t}=\frac{C_{j}^{k+1}-C_{j}^{k}}{\Delta t}+O(\Delta t)
$$

and

$$
\frac{\partial C}{\partial z}=\frac{C_{j+1}^{k}-C_{j}^{k}}{h}+O(h) \text {. }
$$

In order to compute the Caputo fractional derivative, we give the following algorithm [14]. For interval 
$x_{n}=n h: n=0,1,2, \ldots, n$ where $h=X / N$,

$$
\begin{aligned}
& { }_{0}^{C} D_{x}^{\alpha} y_{n}(h)=\left[h^{-\alpha} \Gamma(2-\alpha)\right] \\
& \left.\sum_{n=0}^{N} a_{n, N}() y_{N-n}-\sum_{k=0}^{\lfloor\alpha\rfloor} N,-n{ }^{k} h^{k} \quad k \quad y_{0^{+}}^{(k)}\right)
\end{aligned}
$$

using the quadrature weights

$$
a_{n, N}=\left\{\begin{array}{cc}
1 & n=0 \\
(n+1)^{1-\alpha}-2 n^{1-\alpha}+(n-1)^{1-\alpha} & 0<n<N \\
(1-\alpha) N^{-\alpha}-N^{1-\alpha}+(N-1)^{1-\alpha} & n=N
\end{array}\right.
$$

$$
{ }_{0}^{C} D_{x}^{\alpha} y(x)={ }_{0}^{C} D_{x}^{\alpha} y_{n}(h)+O\left(h^{2-\alpha}\right)
$$

\section{DISCUSSIONS}

Using the properties

$$
{ }_{0}^{C} D_{x}^{\alpha} x^{k}=0, \quad k=0,1, \ldots,\lfloor\alpha\rfloor,
$$

we can obtain the steady state approximation of the problem

$$
\begin{gathered}
0={ }_{0}^{C} D_{x}^{\alpha} c(x, t), \quad 0 \leq x \leq L(t), t>0, \\
(21) \\
c(0, t)=C_{0}, \quad t>0, \\
(22) \\
c(L(t), t)=0, \quad t>0, \\
(23) \\
L(0)=0, \quad(L(t)=m t) .
\end{gathered}
$$

The steady state solution is

$$
c(x, t)=1-x / L(t) .
$$

In Fig. 2 , the concentration $C(z, t)$ versus $z$ with different time $t$ is given and in Fig. 3, the concentration $C(x, t)$ versus $x$ at different time $t$ is given. The straight lines in Fig. 3 are the steady state approximation. If the area is fixed, we know that the long time behavior of the solution is similar to the steady state solution. However, due to the moving boundary, we can see from Fig. 3 that the early time solution is similar to the steady state approximation.

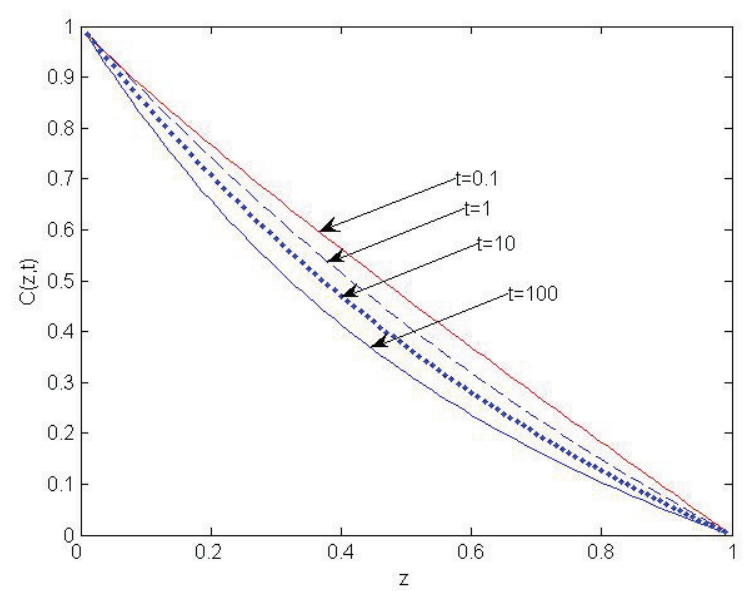

Figure 2. The profile of the concentration $\mathrm{C}(\mathrm{z}, \mathrm{t})$ at time $t=0.1, t=1, t=10$ and $t=100$.

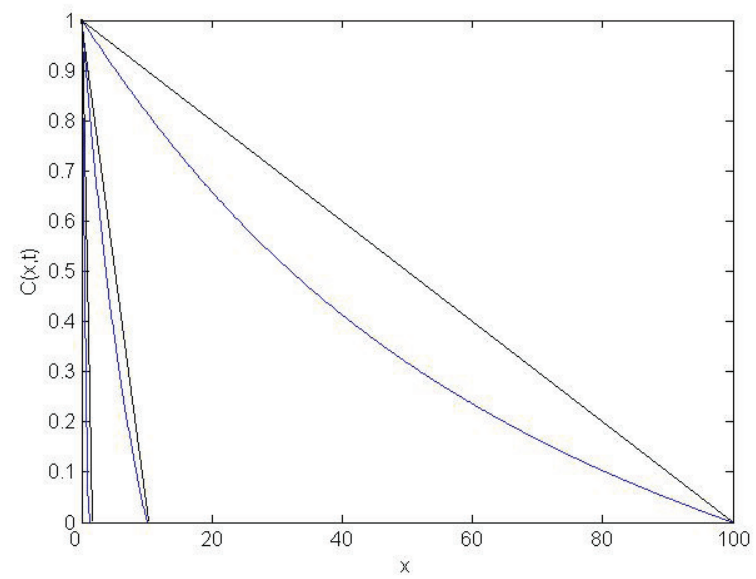

Figure 3. The solution of the concentration $\mathrm{C}(\mathrm{x}, \mathrm{t})$ at time $t=1$, $t=10$ and $t=100$.. The straight lines are the steady state approximation of the problem.

\section{Conclusions}

In this paper, the diffusion process in a variable domain is considered. The moving boundary problem is reduced to a fixed one by letting $z=x / L(t)$. Due to the complexity of the calculus of the fractional derivatives, a numerical method is used. To study the properties of the system, the steady state solution of the problem is also given.

\section{REFERENCES}

[1] A.A. Kilbas, H.M. Srivastava, J.J. Trujillo, Theory and Applications of Fractional Differential Equations, Amsterdam: Elsevier, 2006.

[2] I. Podlubny, Fractional Differential Equations, New York: Academic Press, 1999.

[3] A.S. Chaves, A fractional diffusion equation to describe Lévy flights, Phys. Lett. A, vol. 239, pp. 1316, 1998. 
[4] P. Paradisi, R. Cesari, F. Mainardi, A. Maurizi and F. Tampieri, A generalized Fick's law to describe non-local transport effects, Physics and Chemistry of the Earth, vol. 26, pp. 275-279, 2001.

[5] K. Dierhelm, N.J. Ford, A.D. Freed and Y. Luchko, Algorithms for the fractional calculus: A selection of numerical methods, Comput. Methods Appl. Mech. Engrg, vol. 194, pp. 743-773, 2005.

[6] K. Diethelm, N.J. Ford, A.D. Freed, A predictorcorrector approach for the numerical solution of fractional differential equations, Nonlinear Dyn., vol. 29, pp. 3-22, 2002.

[7] K. Diethelm, N.J. Ford, A.D. Freed, Detailed error analysis for a fractional Adams method. Numer. Algorithms, vol. 36, pp. 31-52, 2004.

[8] N.J. Ford and A.C. Simpson, The numerical solution of fractional differential equations: Speed versus accuracy, Numerical Algorithms, vol. 26, pp. 333-346, 2001

[9] W.H. Deng, Short memory principle and a predictor-corrector approach for fractional differential equations, J. Comput. Appl. Math. Vol. 206, pp. 174$188,2006$.
[10] X.X. Li, Numerical solution of fractional differential equations using cubic B-spline wavelet collocation method, Commun Nonlinear Sci Numer Simulat, vol. 17, pp. 3934-3946, 2012.

[11] J.Y. Liu and M.Y. Xu, Some exact solutions to Stefan problems with fractional differential equations, J. Math, Anal. Appl., vol. 351, pp. 536-542, 2009.

[12] X.C. Li, M.Y. Xu, S.W. Wang, Analytical solutions to the moving boundary problems with space-time-fractional derivatives in drug release devices, Journal of Physics A: Mathematical and Theoretical, vol. 40, pp. 12131-12141, 2007.

[13] X.C. Li, M.Y. Xu, S.W. Wang, Scale-invariant solutions to partial differential equations of fractional order with a moving boundary condition, Journal of Physics A: Mathematical and Theoretical, vol. 41, pp. $155202,2008$.

[14] K. Diethelm, N.J. Ford, A.D. Freed and YU. Luchko, Algorithms for the fractional calculus: A selection of numerical methods, Comput. Methods Appl. Mech. Engrg, vol. 194, pp. 743-773, 2005. 